\title{
Virtual Bulletin Board using Man-Machine Interface (MMI) for Authorized Users
}

\author{
P. Selvi Rajendran ${ }^{1 *}$ and L. Rajendran ${ }^{2}$ \\ 'Department of Computer Science and Engineering, Hindustan Institute of Technology and Science, \\ Chennai -603103, Tamil Nadu, India; p.selvirajendran@gmail.com \\ ${ }^{2}$ Tamil Nadu Veterinary and Animal Sciences University, Chennai - 600051, \\ Tamil Nadu, India; rajendranlak@yahoo.com
}

\begin{abstract}
Objectives: 'Notice board' is most important thing in every institution and public places like bus stops, railway stations and parks. However conveying various notifications day by day is an extremely difficult strategy. This study focuses on advanced notice board called Virtual Bulletin Board (VBB). Methods/Findings: Virtual bulletin Board (VBB) follows the novel procedure to obtain the data for the users. The approved users only can see the content of the VBB. The user can communicate with Board by free hand using MMI. The MMI (Man-Machine Interface) is an integrated interface system that links a network of smart devices together, and allows users to communicate with the physical objects using hand gestures. The user wears a smart glass which shows the user interface in an augmented reality view. Application: The Hand signals are identified by the smart glass and after recognize the correct hand gesture input, VBB will communicate with the associated smart devices to carry out the designated operations. VBB provides common inter-device operations such as file transfer, printing, zooming and touch screen based operations.
\end{abstract}

Keywords: Augmented Reality, Bulletin Board, Hand Gestures, Notice Board, Virtual Board

\section{Introduction}

A 'Notice Board' is an area in which people are able to leave public information, for example, to promote items to buy or sell, make announcements about events, or to providethe information to public. Noticeboardsare usually created with a material such as cork to allow inclusion and also elimination of paper information or it may be put on electronic gadgets like computers, phones. So people can leave and erase messages for other people to read and see. In any College there is a lot of wall notice boards placed on different location where by people with their announcements type on a sheet of paper and place it on the notice boards for other people to read through while passing through those notice boards. Hence there is plenty of piling up of papers on account of negligence of person who put them and no one person who is responsible for eliminating it. Furthermore, the announcement may not reach again to the people who are planned. Hence there may be a lot of stress on the person who are handling to post the notices by ensuring that the announcements are put all over the place, all of these comes mainly because the software is not used in this context. So, people may place and also remove information for others to read and see. The primary goal of this paper would be to make information spreading much simpler in a paperless community as the world tends to adapt into that type of communication to build the Notice Board service as an application, to be used in university administration and public announcement. This article concerns the implementation of VBB to the university administration system. The colleges which includes various departmental notice boards along with

*Author for correspondence 
other related notice boards. This work typically intends to function as a support system for the existing method in which notices have been put up in the VBB web application.

The problems faced by wooden notice board could be well resolved by the implementation of Virtual Bulletin Board application that brings an advanced means of passing notices around in the college in a much easier and efficient way. In this system the authorized user can upload the notices of different categories and departments which is recommended and approved by the higher authorities. On approval, notices can be published in the VBB. Respective viewer may access the content virtually and will make best use out of it. If the user wants to know more about the information received, they can open the file which is transferred from VBB.

\section{Literature Survey}

The existing notice board system used in the institution works on the basis of manual updating method. An Online Announcement Displaying System (OADS) has been developed by Semakuwaet al. ${ }^{1}$ wherein the research instruments such as observation and judgmental techniques were used to get the updates.

In ${ }^{2}$ has developed wireless Digital Notice Board Using GSM Technology. They implemented the system in such a way that it can display message from authorized user sends to GSM module which is located on the notice board. The GSM module which is located at Digital notice board receives the message from authorized user and displayed on notice board which is situated at remote location, at same time this message will be sent to different user's mobile numbers that are stored in microcontroller memory.

$\mathrm{In}^{3}$ developed the e-notice board to provide the access to notices and articles quickly not only within the college premises, also wherever and whenever they need to know. The major strength of the Electronic Notice Board developed, which is an online web application is that, its usability is fully capable of passing relevant notices and announcements, and keeping the users updated from time to time. The user is kept updated each time the E-Notice Board is uploaded based on their preferences with respect to the departments and categories through a SMS. Also the users can view the notices and articles anytime and from anywhere by opening the web application.

The Virtual bulletin Board is an interesting new innovative way to obtain information for the customers, staffs and students. In addition, the information can be accessed by the authorized person only. The user can interact with Board by free hand using MMI. The OADS and e-notice board are working in online only. The wireless Digital notice board can't support for online. The proposed VBB will be working in both online and offline modes.

The Man-Machine Interface (MMI) is a built-in interface system that connects a network of smart devices with each other, and it allows the users to communicate with the VBB using hand gestures. The user wears a smart glass which displays the user interface in an augmented reality view. Hand gestures are captured by the smart glass and upon recognizing the correct gesture input, VBB will communicate with the associated smart devices to complete the specified operations. VBB facilitates popular inter-device operations such as file transfer, printing as well as device pairing.

\section{System Design}

Virtual Bulletin Board is an augmented reality application which requires users to work with wearable equipment such as smart glass. The Smart glasses are configured with cameras, processors, network functionality which enables it to show augmented views to the users. All of these features are usually necessary for the VBB strategy. Since the user is wearing the smart glass, the camera will capture the scene of the adjacent physical environment. The camera recognizes the smart devices in the environment as well as keeps track of their locations according to the details on the corresponding markers. The camera on the smart glass also understands the user hand gestures which allow the user to communicate with objects by freehand manner. The smart glass also provides users with an augmented reality view, which overlays information and interface neatly over the real world.

Designing a VBB takes the consideration of the following:

(i) Physical affordance, which can be access from the user's electronic devices.

(ii) To introduce the virtual interface for the users.

(iii) To provide the communication path for users to bridge the virtual interface and the physical objects.

The Interactive virtual bulletin Board (VBB) can be used in two different modes. The modes of operation are:

a) Virtual mode: When the VBB is in virtual mode which means the user wears the AR glass and attempts 
to access the contents of VBB through MMI. It allows the user to access the contents of the notice board using hand gesture. VBB integrates multiple hand gestures to support several types of freehand interaction. Figure 1 shows the hand gestures which are used for interface interaction.
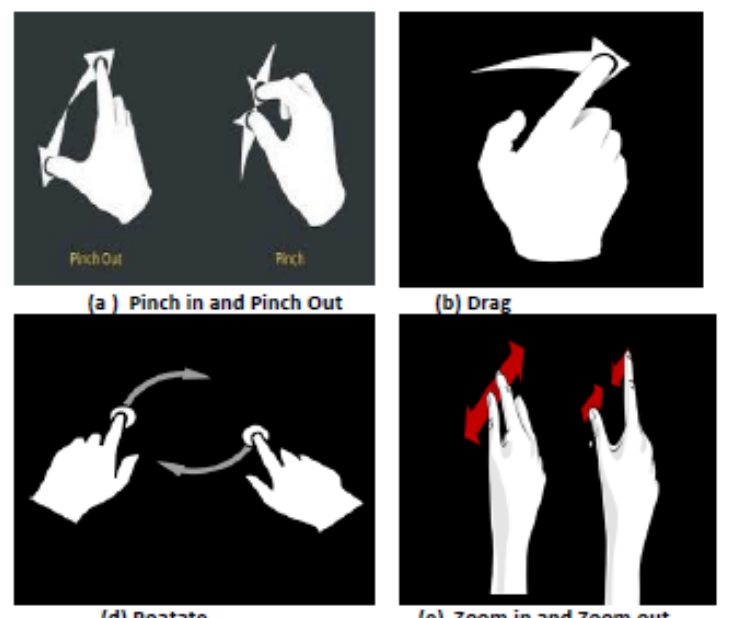

Figure 1. The supported hand gesture for VBB.

b) Non Virtual Mode: The VBB will be act as an electronic notice board. In this context, the viewer or user is connected to the E-notice board through internet and checks for updated notice and also has the privilege to download the updated notice. All the notices are stored in and retrieved from the database.

Pick Operation: A Pinch gesture is interpreted as pick operation. The select operation needs to be carried out on a menu item otherwise it will be invalid.

Drop Operation: An un-pinch gesture plays the role of dropping the picked item. A pick operation need to always end with a drop operation.

Drag Operation: This operation is triggered when a directional gesture is carried out with a pick operation. The selected item is move together with directional gesture in the event that only a single drag operation is offered. The drag interaction ends at any time when a drop operation occurs.

Rotate Operation: A joined operation of a couple of pinch gestures that move reversely along the $\mathrm{Y}$ direction on screen space. The interaction is used to spin Relative distance is mapped as rotation angle. Rotation operation stops whenever either or both pinch gestures end.

Zoom: A combination of a pair of drag operations carried out on the same item. Relative movement is moved to scale up and down the item size. As being one or both of the drag operations terminate, the zoom operation ends.

\section{Prototype Development}

The system Implementation needs technologies from the aspects of object tracking and hand gesture identification. The physical market-based approach can be used for physical object tracking. A pair of distinct strategies which includes double-threshold algorithm, and pre-trained SVM with Bag-Of-Words model, is attempted together with assessed for pinch gesture detection.

Figure 2 displays the process work-flow. The integrated camera on AR Glass usually takes live videos of the scenarios around users, which are streamed for image analysis of object tracking and hand recognition. The method extracts object information of IP address and object type from QR code, which can be used for physical support recognition as well as network communication. Virtual camera is updated as per the real camera pose to render 3D menus from corresponding viewpoint, creating virtual menus associate with surfaces of physical objects. Menus are made as AR overlays to blend with video stream, and then displayed on Google Glass display prism.

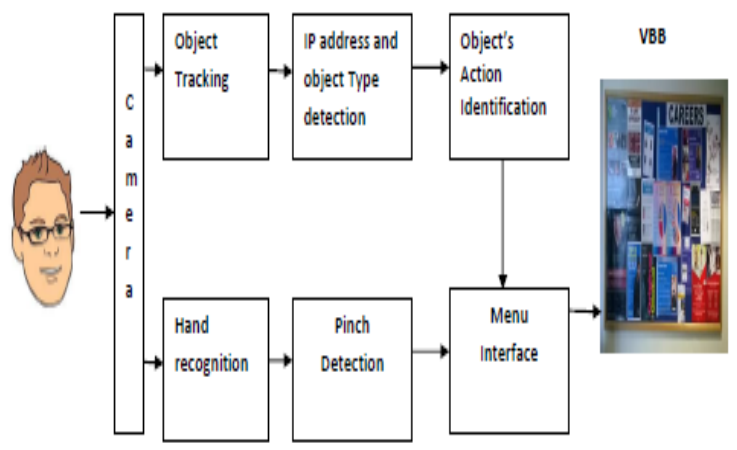

Figure 2. System Architecture of VBB.

Hand gestures are taken out from video stream using a modified inexpensive technique according to the method ${ }^{4}$. The contours are then filtered making use of double thresholds to find out pinch gestures. Users have the ability to communicate with physical objects by way of virtual menus using pinch gestures, which are usually interpreted as socket to carry out communication between physical objects via underlying network. 


\subsection{Object Tracking}

The object tracking consists of in identifying object and also analyzing spatial relationship between objects and users. The marker-based baseline has been applied to do this work, which may be normally more precise and also robust to image distortion and illumination variation ${ }^{5}$. Within this system QR codes are being used as fiducial markers to encode added information for knowing object features. The object information which includes IP addresses and object type is encoded into QR code in advance using the qr code tool

\subsection{Hand Gesture Recognition}

This Approach follows Wilson's vision-based technique to recognize single as well as two-hand pinching gestures, with various enhancements in our technique ${ }^{4}$. The initial step would be to differentiate hands and background using the well-studied segmentation and connected components techniques in computer vision community. Since the background is continually darker when compared with the foreground hands, the approach decides to separate the background from the image.

On the other hand, we extract the foreground hands from the image by applying hand skin color as reference color.

Our method adopts contour size to filter out the incorrect contours. Any specific inner contour with size outside of provided bounds is thrown away. The bonds are identified by the acceptance and elimination curves.

The pinch gesture identification depends entirely on the hole between thumb and index fingers. The approach fails if the whole background is occluded by other curled fingers. It neither does work when the hole is unseen to the camera, such as the thumb and index fingers getting horizontally coplanar. We notice to be a reasonable compromise for freehand communication without device intrusion

\section{Evaluation and Experimentation}

Many experiments have been created to assess the system from different aspects. Participants were asked to carry out the experiments. Their task completion time and also failed trials were calculated and compared. A Technology
Acceptance Model (TAM) $\stackrel{6,7}{ }$ was followed to assess user practical experience.

There are three tasks have been created in connected with selected physical actions which can be carried out on VBB. The participants are equipped with AR glass and mobile or Tab. The computers had installed instant messaging software Skype 4 and share tool Dropbox 5 for transferring as well as sharing document. Many accounts have been created earlier to use the software. Printers and electronic gadgets which are attached with the notice board were running in order that participants could not have to login or turn on devices.

Ex 1: The initial task is to Copying documents between VBB (in Virtual mode) and Mobile. First Participants needed to copy specified documents from notice board in manual operation like take picture by mobile camera. In $\mathrm{VBB}$, participants were required to carry out pick, drag, and drop operations with their hands to finish document copy. Rotate operation may be needed when the specified document items are folded in menus.

Ex 2: In the following task, the Copying documents between Non Virtual mode of VBB and Mobile are taking into consideration. The participants were asked to transfer the contents from VBB to mobile using Bluetooth or wifi options.

Ex 3: Detection rate and failure rate of gesture identification is taken into consideration for third task. Contours of hand section are then extracted. The biggest one is the outer contour of the hand section as illustrated in Figures 3-4. Any inner contour shows a hole in the hand section. General illumination along with other non-pinch gestures may additionally generate the inner contours.

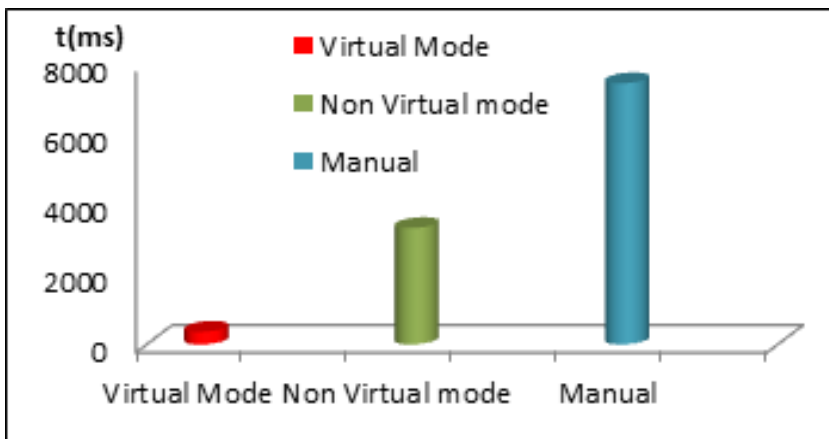

Figure 3. Comparison of task competition time of experiments by using VBB and traditional methods. 

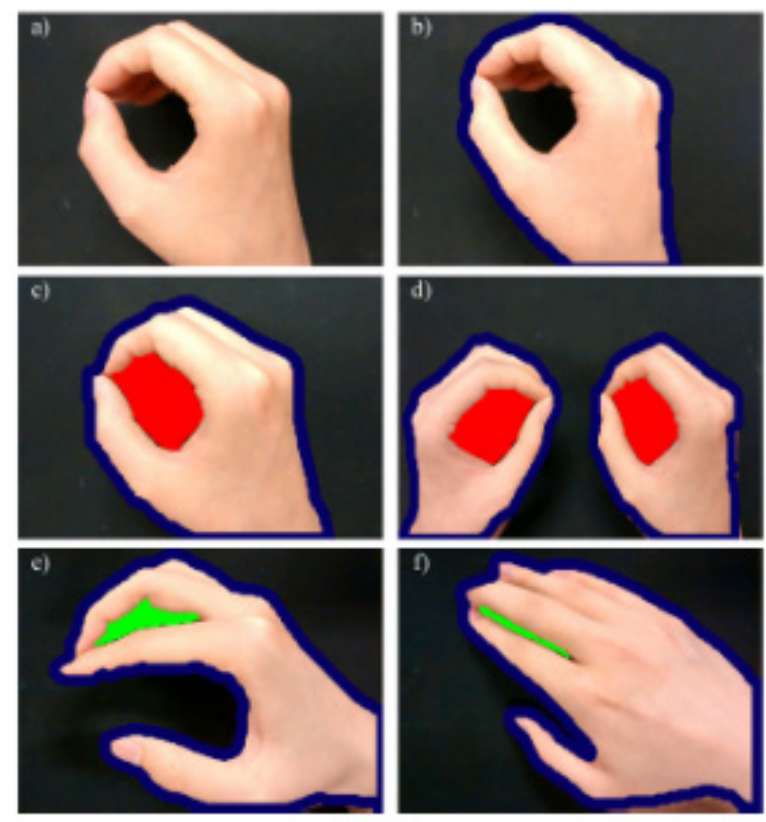

Figure 4. The pinch gesture detection. a) A typical pinch gesture; b) outer contour of hand is extracted; c) and d): red closed regions are recognized as pinch holes; e) and f): green closed regions are eliminated as non-pinch holes.

\section{Results and Discussion}

Figure 3 compares the participants' task completion time using VBB (virtual mode) system and other traditional techniques like document copy via USB devices and Skype, document share through Dropbox and using other networking features. The time expense of VBB is significantly a lot less than other traditional methods in every task. Particularly, it is taken $360 \mathrm{~ms}$ (Red bar) for move the contents which is nearly 4 to 7 times much faster respectively when compared with transferring on Skype (Green bar, Non Virtual mode $=3317 \mathrm{~ms}$ ) and USB flash copy. The manual transfer will be done through observing the content manually or photo image taken by mobile. It is taken $7442 \mathrm{~ms}$ for processing manually in Ex1.

Making use of USB flash copy involves plugging in, plugging out, and copying documents between USB devices and mobiles. VBB simplifies the manipulations without direct physical touch. For document printing VBB takes $\mathrm{VBB}=380 \mathrm{~ms}$ also it eliminates operations of clicking \print..." menu item and choosing specified printer from printer list needed by traditional method (10242 ms) It was clear that a few participants referenced to task cards when they selected a printer from configuration panel.
VBB $(\mathrm{M}=8735 \mathrm{~ms}, \mathrm{VBB}=307 \mathrm{~ms})$ can also be more effective in document sharing in comparison with Dropbox sharing $(\mathrm{M}=13502 \mathrm{~ms}, \mathrm{VBB}=1127 \mathrm{~ms})$, as it would not require verifying target user's Dropbox account, that may be normally not the same as target computer's account.

Almost all pinch gestures identification will pass when the threshold is small, as shown by the acceptance curve that recognition accuracy decreases when the threshold goes up. A lower bound 0.6 and upper bound value 0.8 has been set to achieve over $85 \%$ accuracy of both pinch gesture detection and non-pinch gesture removal. The approach will work in both one and two-hand pinch gesture identification as shown in Figure 4. It is usually robust to remove other non-pinch gestures. Figure 4. show two gestures with holes in hand sections, but they are removed in our approach as the pinch holes are small compared with the given lower bound value. In practice, it can be applicable to uncheck the upper bound as result set of a small upper bound continually consists of result of a large upper bound. However, size of pinch hole is constrained to hand anatomy. An acceptable upper bound ensures excluding outliers.

\section{Conclusion}

Virtual bulletin Board (VBB) provides a decentralized user interface system for manipulation between the physical and digital worlds. User interface and communication are carefully designed to manage free-hand user experience. This technique is evaluated from both aspects of technological and user experience. By communicating with objects at a high-level of physical affordance instead of fundamental electronic counterparts, this approach supports users carry out operations in much comfortable as well as natural way. It has been shown to be helpful for simple and frequently-performed manipulations with associated digital devices in closed environments.

In our experiments, it was clear that lot of failures were occurred due to hand gesture detection. The color-based method is selected to relieve computational cost, but it is not correct enough in backgrounds with similar color of hand skin. In future we decide to incorporate depth and visual cameras to enhance the accuracy. Another problem is the limitation of weak computational capability and minimal power capacity of AR Glass. The approach has been verified to improve both real-time performance and runtime sustainability. 


\section{References}

1. Semakuwa SK, Rashid FU, Fungo DC, Mbwana RY. Migrant from on wall notice-board to an online announcement displaying system for Tanzanian, college's. International Journal of Research in Computer Applications and Robotics. 2014; 2(9): 88-95.

2. Gurav RK, Jagtap R. Wireless digital notice board using GSM technology. International Research Journal of Engineering and Technology. 2015; 2(9).

3. Anushree SP, Bhat DV, Moonisha GA, Venkatesh UC. Electronic notice board for professional college. International Journal of Science, Engineering and Technology Research. 2014; 3(6).
4. Wilson D. Robust computer vision-based detection of pinching for one and two-handed gesture input. Proceedings of UIST; 2006. p. 255-8. https://doi.org/10.1145/1166253.1166292

5. Neumann U. Natural feature tracking for augmented reality. IEEE Transactions on Multimedia. 1999; 1(1): 53-64. https://doi.org/10.1109/6046.748171

6. Haugstvedt AC, Krogstie J. Mobile augmented reality for cultural heritage: A technology acceptance study. IEEE International Symposium on Mixed and Augmented Reality; 2012.p.247-55.https://doi.org/10.1109/ISMAR.2012.6402563

7. Ren G, Neill EO. 3d selection with freehand gesture. Computers and Graphics. 2013; 37(3):101-20. https://doi. org/10.1016/j.cag.2012.12.006 